\title{
Genes Frequently Coexpressed with Hoxc8 Provide Insight into the Discovery of Target Genes
}

\author{
Ruthala Kalyani, ${ }^{1,3,4}$, Ji-Yeon Lee ${ }^{1,4}$, Hyehyun Min ${ }^{1,4}$, Heejei Yoon ${ }^{2, *}$, and Myoung Hee Kim ${ }^{1, *}$
}

\begin{abstract}
Identifying Hoxc8 target genes is at the crux of understanding the Hoxc8-mediated regulatory networks underlying its roles during development. However, identification of these genes remains difficult due to intrinsic factors of Hoxc8, such as Iow DNA binding specificity, contextdependent regulation, and unknown cofactors. Therefore, as an alternative, the present study attempted to test whether the roles of Hoxc8 could be inferred by simply analyzing genes frequently coexpressed with Hoxc8, and whether these genes include putative target genes. Using archived gene expression datasets in which Hoxc8 was differentially expressed, we identified a total of 567 genes that were positively coexpressed with Hoxc8 in at least four out of eight datasets. Among these, 23 genes were coexpressed in six datasets. Gene sets associated with extracellular matrix and cell adhesion were most significantly enriched, followed by gene sets for skeletal system development, morphogenesis, cell motility, and transcriptional regulation. In particular, transcriptional regulators, including paralogs of Hoxc8, known Hox co-factors, and transcriptional remodeling factors were enriched. We randomly selected Adam19, Ptpn13, Prkd1, Tgfbi, and Aldh1a3, and validated their coexpression in mouse embryonic tissues and cell lines following TGF- $\beta 2$ treatment or ectopic Hoxc8 expression. Except for Aldh1a3, all genes showed concordant expression with that of Hoxc8, suggesting that the coexpressed genes might include direct or indirect target genes. Collectively, we suggest that the coexpressed genes provide a resource for constructing Hoxc8mediated regulatory networks.
\end{abstract}

\section{INTRODUCTION}

Hoxc8 is a homeodomain transcription factor that regulates pattern formation, cell migration, and differentiation (Lei et al., 2005; Pearson et al., 2005). Loss- or gain-of-function studies have suggested that Hoxc8 is essential for skeletal pattern formation, hematopoiesis, and cartilage differentiation during embryogenesis (Kruger and Kappen, 2010; Le Mouellic et al,. 1992; Shimamoto et al., 1999; Tiret et al., 1998; Yueh et al., 1998). To further elucidate the underlying mechanisms of developmental defects caused by Hoxc 8 mutation, it is necessary to identify the transcriptional target genes of Hoxc8. To date, several target genes have been identified, and several preliminary Hoxc8-mediated regulatory networks have been proposed Hoxc8 directly downregulates Opn, Zac1, Ncam, and Pedf, and upregulates Cdh11 in mouse embryonic fibroblast cells (Lei et al., 2005; 2006). We also previously identified Pcna, ZNF804A, and $M g / 1$ as Hoxc8 direct target genes in mouse embryonic fibroblast cells (Chung et al., 2010; Min et al., 2010; Ruthala et al., 2011). In another study, although 15 of 21 target genes were upregulated by Hox genes (Pearson et al., 2005), more than half of the known Hoxc8 target genes were downregulated by Hoxc8. This bias in Hoxc8 regulation patterns might result from use of a single cell type, mouse embryonic fibroblast cells at specific developmental stage in vitro. To more accurately reflect in vivo Hoxc8 transcriptional specificity, more target genes need to be identified in diverse cell types and tissues throughout the stages of embryonic development.

Several characteristics of Hoxc8 make target gene identification difficult. First, it has low DNA binding sequence specificity. Hoxc8 proteins bind as monomers or multimers to specific sequence motifs (TAAT/ATTA, TTAT, and ATAA) in their target genes (Lei et al., 2006). However, other Hox proteins can potentially bind to the same elements, and the DNA-binding specificities are modified through interactions with cofactors, such as $\mathrm{Pbx}$ and Meis (Ladam and Sagerström, 2014, Mann, 1995; Mann and Chan, 1996; Mann and Affolter, 1998; Moens and Selleri, 2006;). Second, little is known about Hoxc8 cofactors. Interaction of HOXC8 with PBX1 has been reported in prostate cancer cells (Kikugawa et al., 2006) but in no other tissues. Interestingly, Hoxc8 interacts with Smad1 during osteoblast differentiation (Hullinger et al., 2001, Shi et al., 1999; Yang et al., 2000). This indicates that other unknown factors, even those without a homeodomain like Smads, might interact with Hoxc8 in vivo. Third, Hox members act as both transcriptional activators and repressors, depending on their cellular context (Pearson et al., 2005). Therefore, careful experimental designs, with

Keywords: coexpressed genes, Hox genes, in silico analysis, pattern formation, vertebrate development

elSSN: 0219-1032

(c) The Korean Society for Molecular and Cellular Biology. All rights reserved.

(c) This is an open-access article distributed under the terms of the Creative Commons Attribution-NonCommercial-ShareAlike 3.0 Unported License. To view a copy of this license, visit http://creativecommons.org/licenses/by-nc-sa/3.0/. 
attention to cell types, developmental stage, time, and location, are necessary to validate candidate genes.

In an attempt to identify more Hoxc8 target genes, we analyzed eight archived microarray datasets generated from diverse cell types and tissues throughout mouse embryonic development. We found that Hoxc8 is often coexpressed with several transcription factors, including known cofactors $P b x 1$ and Meis, and genes related to extracellular matrix (ECM) or organization. We then randomly selected five genes and assessed their likelihood of being Hoxc8 target genes.

\section{MATERIALS AND METHODS}

\section{Gene expression data analysis}

To identify genes with expression patterns similar to those of Hoxc8 during mouse embryonic development, we used gene expression profiles from the GEO database (http://www.ncbi. nlm.nih.gov/geo/). We analyzed eight datasets (Table 1) and assessed the similarity of each gene expression pattern to that of Hoxc8 based on Pearson's correlation coefficient ( $r$-value $>$ 0.75 ) and $t$-test ( $p$-value $<0.001)$. For the $t$-test, we grouped samples based on differential Hoxc8 expression, as shown in Table 1. We selected genes with r-values greater than 0.75 in at least four out of the eight datasets. Genes with Pearson's correlation coefficients greater than 0.75 in at least four out of the eight datasets were subjected to gene set analysis with the Database for Annotation, Visualization, and Integrated Discovery (DAVID) (http://david.abcc.ncifcrf.gov/home.jsp) program. Gene Ontology (GO) annotations with an enrichment score > 1.0 , p-value $<0.01$, and false discovery rate (FDR) $\leq 5 \%$ were considered biologically significant. We used the rVista sequence analyzer (http://zpicture.dcode.org/) to search for Hox consensus binding elements present in the $5 \mathrm{~kb}$ promoter, first exon, and first intron of putative target genes. The gene network was analyzed with Ingenuity Pathway Analysis (IPA) (http://www.ingenuity.com/products/ipa).

\section{Animal preparation}

To obtain E14.5 embryos, male and female ICR mice were caged together for mating at around $6 \mathrm{pm}$. The next morning, when vaginal plugs were present, was defined as 0.5 days post-coitum (dpc) or as an E0.5 embryo. After 14 days, the pregnant female mice were sacrificed, and the E14.5 embryos were extracted. The maternal and extra-embryonic tissues, cervical region, internal organs, tail bud, and limbs were removed. The embryos were divided into three parts: brain, trunk anterior (somites 12-23), and trunk posterior (somites 24-41). Total RNA was isolated from each part. This study was carried out in strict accordance with the recommendations in the Guide for the Institutional Animal Care and Use Committee of Yonsei University College of Medicine. The protocol for obtaining embryonic samples was approved by the Committee on Animal Research at Yonsei University College of Medicine (permit number 2013-0174-1).

\section{Cell culture and transfection}

Three cell lines, MC3T3-E1, NIH3T3, and C3H10T1/2, were cultured in Dulbecco's modified Eagles medium (WelGENE Inc., Korea) supplemented with $10 \%$ fetal bovine serum (FBS; WelGENE Inc., Korea) and $100 \mu \mathrm{g} / \mathrm{ml}$ penicillin-streptomycin (WelGENE Inc., Korea) at $37^{\circ} \mathrm{C}$ inside a $5 \% \mathrm{CO}_{2}$ and $95 \%$ humidified incubator. The construction of the pcDNA3.1-Hoxc8 plasmid, harboring the murine Hoxc8 gene, has been previously described (Kwon et al., 2003). pcDNA3.1-Hoxc8 or pcDNA3.1 empty vector was transfected into MC3T3-E1 cells using Lipofectamine $2000^{\mathrm{TM}}$ reagent (Invitrogen, USA), as indicated by the manufacturer. To establish stable cell lines expressing Hoxc8, NIH3T3 and C3H10T1/2 cells were transfected with Lipofectamine $2000^{\mathrm{TM}}$ reagent and pcDNA3.1-Hoxc8 or control empty vector and selected in culture media containing 500 $\mu \mathrm{g} / \mathrm{ml}$ G418 antibiotic (Invitrogen, USA). The media was changed every three days. Cells were subcultured when the cells reached $90 \%$ confluence.

MC3T3-E1 cells were seeded into 12-well dishes at $1 \times 10^{5}$ cells/well. After an overnight incubation, the media was replaced with fresh media supplemented with $10 \mathrm{ng} / \mathrm{ml}$ of TGF- $\beta 2$ (R\&D Systems, Inc., USA). Cells were harvested at time points $0 \mathrm{~h}, 6 \mathrm{~h}$, and $12 \mathrm{~h}$ after treatment with TGF- $\beta 2$, and total RNA was isolated using Trizol reagent (Invitrogen, USA).

Knockdown of Hoxc8 with short interfering RNA (siRNA) The sequences of the Hoxc8 and control siRNAs are as follows: Hoxc8 sense, 5'-AGA CGC CUC CAA AUU CUA UTT-3', Hoxc8 antisense, 5'-AUA GAA UUU GGA GGC GUC UTT-3', control sense, 5'-AUG AAC GUG AAU UGC UCA ATT-3', and control antisense, 5'-UUG AGC AAU UCA CGU UCA UTT-3' (Samchully Pharm Co., Ltd., Korea). MC3T3-E1 cells were seeded into 12-well dishes $\left(1 \times 10^{5}\right.$ cells/well $)$ and incubated overnight. Then, $100 \mathrm{nM}$ siRNA (final concentration) was transfected into the cells using HiPerfect transfection reagent (Qiagen, Germany), according to the manufacturer's instructions. Cells were harvested $60 \mathrm{~h}$ after transfection, followed by isolation of total RNA. All experiments were performed in triplicate and representative examples are shown.

\section{RNA isolation and semi-quantitative RT-PCR}

Total RNA was isolated using Trizol reagent, according to the manufacturer's instructions (Invitrogen, USA). Two micrograms $(\mu \mathrm{g})$ of total RNA was reverse-transcribed with ImProm-IIM Reverse Transcriptase (Promega, USA) and poly $(\mathrm{dT})_{20}$, according to the manufacturer's instructions. Semi-quantitative RT-PCR was performed with hTaq DNA polymerase (Solgent, Korea) using the following thermo cycling conditions: initial denaturation for $5 \mathrm{~min}$ at $95^{\circ} \mathrm{C}$, followed by 30-33 cycles of $94^{\circ} \mathrm{C}$ for $30 \mathrm{~s}$ (denaturation), $58^{\circ} \mathrm{C}$ for $30 \mathrm{~s}$ (annealing), and $72^{\circ} \mathrm{C}$ for $30 \mathrm{~s}$ (polymerization). We determined the highest PCR cycle numbers at which PCR products increased linearly and were detectable on agarose gel. Primer sequences are listed in Supplementary Table S1. The PCR products were analyzed on a $1.5 \%$ agarose gel containing ethidium bromide.

\section{RESULTS}

\section{Hoxc8 was differentially expressed in several gene} expression datasets

To select archived gene expression datasets in which Hoxc8 was differentially expressed, the GEO database was analyzed. We selected datasets by focusing on two criteria: datasets associated with studies on mouse development and cell differentiation, as well as those with greater than two-fold differential Hoxc8 expression under the given experimental conditions, tissue types, or cell types. Eight datasets (GDS2843, GDS2743, GDS1500, GDS2123, GDS2209, GDS2699, GDS2044, and GDS2421) met our criteria (Table 1). For example, Hoxc8 was differentially expressed during mammary gland development (GDS2843; Fig. 1). In addition, when different cell types were compared, Hoxc8 was overexpressed in white adipocytes compared to brown adipocytes (GDS2743), spinal cord com- 
Table 1. Gene expression datasets in which Hoxc8 was differentially expressed

\begin{tabular}{|c|c|c|c|c|c|}
\hline $\begin{array}{l}\text { GEO } \\
\text { Data Set No. }\end{array}$ & Title & Summary & Hoxc8 expression & Opn (r) & $\mathrm{Fzd} 2(\mathrm{r})$ \\
\hline GDS2843 & $\begin{array}{l}\text { Mammary gland } \\
\text { development }\end{array}$ & $\begin{array}{l}\text { Analysis of mammary glands during } \\
\text { pregnancy, lactation, and involution }\end{array}$ & $\begin{array}{l}\text { Hoxc8 was sharply } \\
\text { downregulated } 7 \text { days after } \\
\text { pregnancy and maintained at a } \\
\text { low level throughout lactation } \\
\text { and involution }\end{array}$ & -0.64 & 0.9 \\
\hline GDS2743 & $\begin{array}{l}\text { Brown and white } \\
\text { adipocyte differentiation }\end{array}$ & $\begin{array}{l}\text { Comparison of brown and white } \\
\text { preadipocytes at the undifferentiated and } \\
\text { differentiating stages }\end{array}$ & $\begin{array}{l}\text { Hoxc8 was overexpressed in } \\
\text { white adipocyte compared to } \\
\text { brown adipocyte, irrespective of } \\
\text { differentiation status }\end{array}$ & -0.39 & 0.8 \\
\hline GDS1500 & $\begin{array}{l}\text { Mechanical stress effect } \\
\text { on fibroblasts from } \\
\text { various fetal tissues }\end{array}$ & $\begin{array}{l}\text { Expression profiling of fetal fibroblasts } \\
\text { from tendon, skin, and cornea following } \\
\text { mechanical stimulation by fluid flow. } \\
\text { Fibroblasts isolated from embryos at } 19 \\
\text { days post conception }\end{array}$ & $\begin{array}{l}\text { Hoxc8 was relatively } \\
\text { overexpressed in skin } \\
\text { fibroblasts compared to corneal } \\
\text { or tendon fibroblasts isolated } \\
\text { from embryos at } 19 \text { days } \\
\text { post-conception }\end{array}$ & -0.76 & 0.17 \\
\hline GDS2123 & $\begin{array}{l}\text { Brown fat cell response } \\
\text { to PGC-1alpha and } \\
\text { PGC-1beta deficiency }\end{array}$ & $\begin{array}{l}\text { Analysis of brown fat cells lacking } \\
\text { PGC-1alpha or both PGC-1alpha and } \\
\text { PGC-1beta. PGC-1alpha is required for } \\
\text { the thermogenic function of brown fat } \\
\text { cells, and PGC-1beta is the } \\
\text { closest homolog of PGC-1alpha }\end{array}$ & $\begin{array}{l}\text { Hoxc8 was remarkably } \\
\text { downregulated in brown fat cells } \\
\text { lacking PGC-1alpha or lacking } \\
\text { both PGC-1alpha and } \\
\text { PGC-1beta }\end{array}$ & -0.87 & 0.87 \\
\hline GDS2209 & $\begin{array}{l}\text { Spinal cord and dorsal } \\
\text { root ganglion }\end{array}$ & $\begin{array}{l}\text { Analysis of spinal cord (SC) and dorsal } \\
\text { root ganglion (DRG). The central nervous } \\
\text { system (CNS) comprises the brain and } \\
\text { SC; the peripheral nervous system (PNS) } \\
\text { includes spinal and cranial nerves along } \\
\text { with their associated DRGs }\end{array}$ & $\begin{array}{l}\text { Hoxc8 was upregulated in the } \\
\text { spinal cord (SC), but it was } \\
\text { downregulated in the dorsal root } \\
\text { ganglion (DRG). }\end{array}$ & -0.83 & -0.9 \\
\hline GDS2699 & $\begin{array}{l}\text { Mesenchymal and } \\
\text { epithelial compartments } \\
\text { of the developing } \\
\text { intestine }\end{array}$ & $\begin{array}{l}\text { Analysis of the mesenchymal and } \\
\text { epithelial fractions of the embryonic small } \\
\text { intestine. Crosstalk between the } \\
\text { epithelium and the underlying } \\
\text { mesenchyme is required for intestinal } \\
\text { development }\end{array}$ & $\begin{array}{l}\text { Hoxc8 was upregulated in } \\
\text { mesenchymal cells compared to } \\
\text { the epithelial fractions of the } \\
\text { embryonic small intestine. }\end{array}$ & 0.9 & 0.99 \\
\hline GDS2044 & $\begin{array}{l}\text { DNA demethylation } \\
\text { effect on dermal } \\
\text { fibroblasts }\end{array}$ & $\begin{array}{l}\text { Analysis of primary dermal fibroblasts } \\
\text { treated with the DNA methyltransferase } \\
\text { inhibitor } 5 \text {-aza-2'-deoxycytidine (Aza-dC) } \\
\text { for } 96 \text { hours }\end{array}$ & $\begin{array}{l}\text { Hoxc8 was downregulated in } \\
\text { primary dermal fibroblasts } \\
\text { treated with the DNA } \\
\text { methyltransferase inhibitor 5- } \\
\text { aza-2'-deoxycytidine (Aza-dC). }\end{array}$ & -0.17 & 0.97 \\
\hline GDS2421 & $\begin{array}{l}\text { Fibroblast growth factor } \\
2 \text { effect on embryonic } \\
\text { fibroblast: dose } \\
\text { response }\end{array}$ & $\begin{array}{l}\text { Analysis of embryonic fibroblasts after } \\
\text { treatment with fibroblast growth factor } 2 \\
\text { (FGF2) at } 4,13 \text {, and } 40 \mathrm{ng} / \mathrm{ml} \text { for } 24 \\
\text { hours. FGF2 promotes the self-renewal of } \\
\text { embryonic stem cells (ESCs) }\end{array}$ & $\begin{array}{l}\text { Hoxc8 was downregulated after } \\
\text { treatment with fibroblast growth } \\
\text { factor } 2 \text { (FGF2). FGF2 } \\
\text { promotes the self-renewal of } \\
\text { embryonic stem cells (ESCs) }\end{array}$ & -0.98 & 0.99 \\
\hline
\end{tabular}

pared to dorsal root ganglion (GDS2209), mesenchymal cells compared to epithelial cells (GDS2699), and in skin fibroblasts among fetal corneal, skin, and tendon fibroblasts (GDS1500). Next, we examined the datasets in which the known Hoxc8 target genes Opn and $F z d 2$ were concordantly expressed with Hoxc8 (Table 1). Representative data from the GDS2843 dataset, which originated from the developing mammary gland, are shown in Fig. 1. Therein, the gene expression levels of Opn and $F z d 2$ were negatively and positively correlated with that of Hoxc8, respectively. In most datasets, the expression patterns of $F z d 2$ were highly correlated with Hoxc8 (Table 1) except in the GDS1500 and GDS2209 datasets.

\section{Enriched functions of Hoxc8 coexpressed genes}

To search for genes coexpressed with Hoxc8 in each dataset, we assessed the similarity of gene expression patterns between each gene and Hoxc8 using Pearson's correlation coefficient ( $r$-value $>0.75$ ) and t-test ( $p$-value $<0.001$ ). A total of 567 genes that had r-values greater than 0.75 in at least four of eight datasets were selected (Fig. 2). Among these, 23 genes were found to have r-values greater than 0.75 in six of the eight datasets (Table 2). Among these 23 genes, Ncam1 and Fzd2 
Genes Concordantly Expressed with Hoxc8

Ruthala Kalyani et al.

Table 2. Genes most frequently coexpressed with Hoxc8

\begin{tabular}{|c|c|}
\hline Gene symbol & Name \\
\hline Nfic & Nuclear factor I/C \\
\hline Tgfbi & Transforming growth factor, beta induced \\
\hline Anp32a & Acidic (leucine-rich) nuclear phosphoprotein 32 family, member $A$ \\
\hline Thbs2 & Thrombospondin 2 \\
\hline Fzd2 & Frizzled homolog 2 (Drosophila) \\
\hline Ncam1 & Neural cell adhesion molecule 1 \\
\hline Ldhb & Lactate dehydrogenase B; predicted gene 5514 \\
\hline Sparc & Secreted acidic cysteine rich glycoprotein; similar to Secreted acidic cysteine rich glycoprotein \\
\hline Fbln1 & Fibulin 1 \\
\hline Ift81 & Intraflagellar transport 81 homolog (Chlamydomonas) \\
\hline Timp3 & Tissue inhibitor of metalloproteinase 3 \\
\hline P4ha2 & Procollagen-proline, 2-oxoglutarate 4-dioxygenase (proline 4-hydroxylase), alpha II polypeptide \\
\hline Plat & Plasminogen activator, tissue \\
\hline Tmem45a & Transmembrane protein $45 a$ \\
\hline Mylk & Myosin, light polypeptide kinase \\
\hline Fyn & Fyn proto-oncogene \\
\hline Clasp1 & CLIP associating protein 1 \\
\hline Fbxo21 & F-box protein 21 \\
\hline Pltp & Phospholipid transfer protein \\
\hline Islr & Immunoglobulin superfamily containing leucine-rich repeat \\
\hline Meis1 & Meis homeobox 1 \\
\hline Sspn & Sarcospan \\
\hline Cugbp2 & CUG triplet repeat, RNA-binding protein 2 \\
\hline
\end{tabular}

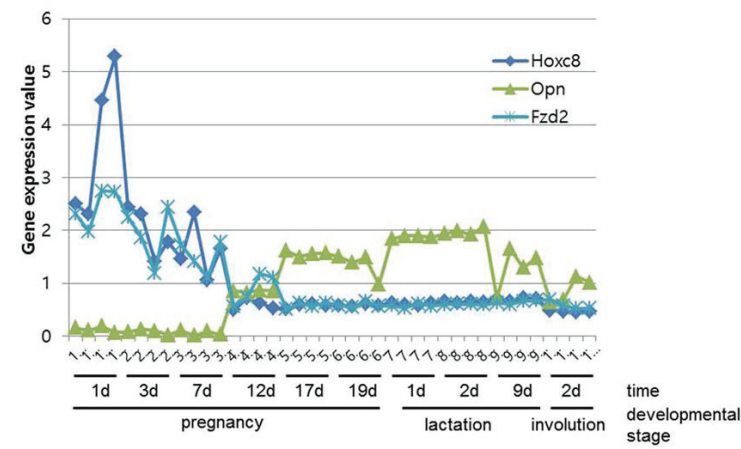

Fig. 1. Correlation of Hoxc8 expression with expression of known downstream genes, $O p n$ and $F z d 2$, during mammary gland development. Gene expression levels of $H o x c 8, O p n$, and Fzd2 at each time point were obtained from the GEO database (GDS2843). For each time point, there were four biological replicates of the microarray experiments. Hoxc8 expression gradually decreased until pregnancy day 7 and was maintained at low levels throughout lactation and involution. Fzd2 exhibited similar behavior to Hoxc8. In contrast, Opn, which is negatively regulated by Hoxc8, was completely repressed until pregnancy day 7 and was then induced and maintained after 12 days of pregnancy.

are already known to be Hoxc8 target genes, and Meis1 is a known Hox co-factor. As the most of the known target genes are associated with ECM or cell adhesion, many of the identified genes were found to be associated with ECM (Isir, immunoglobulin superfamily containing leucine-rich repeat; Timp3; Sparc; Fbln1; Tgfbi, transforming growth factor beta-induced, $68 \mathrm{kDa}$ ) or cell adhesion (Thbs2, thrombospondin 2; Fyn; Mylk, myosin light chain kinase).

To gain insight into the functional characteristics of Hoxc8 coexpressed genes, we performed gene set analysis for 567 genes, using the DAVID program. Genes associated with the ECM were most significantly enriched (Fig. 2 and Supplementary Table S2). In addition to the 23 genes mentioned above, collagen family proteins (Col27a1, Col3a1, Col1a2, Col1a1, Lox, Col11a1, Col5a2, Col5a1, and Col4a5) and proteolysis metallopeptidases (Adam19, Adam9, and Adamts4, -5, and -12) were highly enriched. These genes are closely connected with the Hoxc8 subnetwork (Supplementary Fig. S1). Genes associated with the development of the skeletal system were also highly enriched. Of note, these included six other Hox genes: Hoxa5, Hoxa7, Hoxc6, Hoxc9, Hoxd8, and Hoxd9. These genes are neighboring genes localized next to Hoxc8 and its paralogues located in other clusters, with the exception of the Hoxb cluster. We also performed gene set analysis for genes that were negatively correlated with Hoxc8 at least four times in eight datasets (Supplementary Table S3). Most significantly, the enriched gene set was associated with the generation of precursor metabolites and energy, especially the TCA cycle and localization in the mitochondria.

Interestingly, $17.4 \%$ of the coexpressed genes were transcriptional regulators, such as transcription factors involved in a 
A

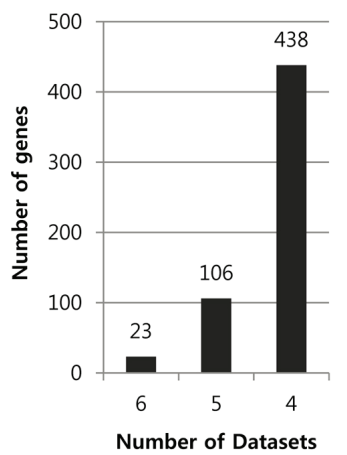

A

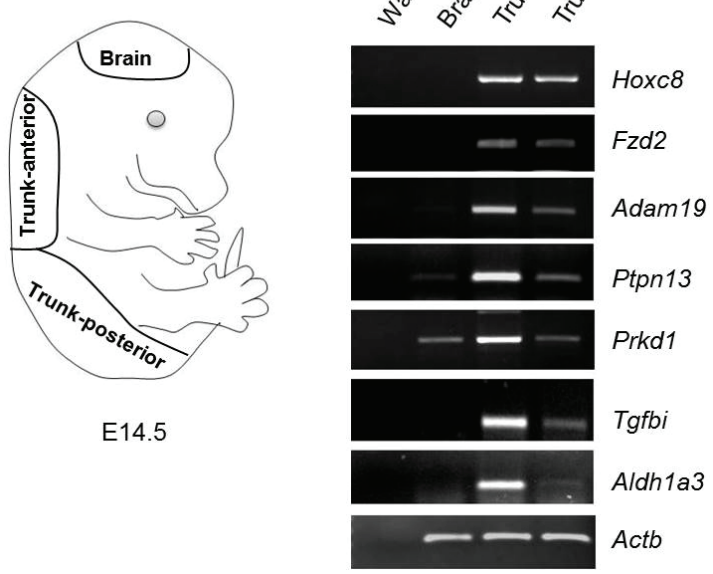

Fig. 3. Endogenous expression patterns of Hoxc8 and its coexpressed genes in mouse embryonic tissues. (A) E14.5 mouse embryos indicating the location of the brain, trunk-anterior, and trunkposterior tissues used for RT-PCR analysis. (B) Semi-quantitative RT-PCR was performed for Hoxc8 and the 5 selected genes. Fzd2 served as a positive control for Hoxc8 expression, and Actb was used as a loading control. "Trunk-ant" and "trunk-pos" indicate the "trunk anterior" and "trunk posterior" regions, respectively. "Water" represents a negative control reactions containing water instead of template DNA.

pattern formation, Hox cofactors, and chromatin remodeling factors. In addition to Meis1, other well-known Hox cofactors, including Meis2, Pbx1, and $\mathrm{Pbx} 3$, belonging to the TALE (Three Amino acid Loop Extension) family, were concordantly expressed in four datasets. Zhx1, Prrx1, Zeb2, Pknox1, and Meox2 also have a homeobox domain, as do members of the TALE family. Zhx1 and Prrx1 are members of the zinc-finger and homeobox protein families, respectively, both of which act as repressors. However, whether they function as Hox cofactors is unknown. Although the tissues or cells used in the gene expression datasets are heterogeneous, gene set analysis of the coexpressed genes seemed to summarize Hoxc8 phenotypes.

\section{Selected genes were endogenously coexpressed with}

Hoxc8

To assess the possibility that the 567 selected genes are coexpressed with $\mathrm{Hoxc} 8$, we selected five genes and compared their expression patterns with that of Hoxc8 in mouse embryos and cell lines. We selected the five following genes showing different coexpression frequencies against Hoxc8: Tgfbi (transforming growth factor, beta induced), Ptpn13 (protein tyrosine phosphatase non-receptor type 13), Prkd1 (protein kinase D1), Adam19 (a disintegrin and metallopeptidase domain 19), and Aldh1a3 (aldehyde dehydrogenase 1a3). Tgfbi was the most frequently coexpressed ( 6 of 8 datasets) gene, while Aldh1a3 was coexpressed in only three out of eight datasets. Prkd1 and Ptpn13 were coexpressed in five, and Adam19 was coexpressed in four of the eight datasets (Supplementary Table S4). These proteins have been implicated in various biological systems, such as ECM and adhesion (Tgfbi and Adam19), phosphate metabolic processes (Ptpn13 and Prkd1), and retinoic acid biosynthetic processes (Aldh1a3).

First, the endogenous expression patterns of these five genes were analyzed in mouse embryos (Fig. 3). E14.5 embryos were dissected into three parts, brain, trunk anterior, and trunk posterior tissues, and then semi-quantitative RT-PCR was performed after isolating total RNA from each tissue. In agreement with previous reports (Kwon et al., 2005; Min et al., 2012; 2013), Hoxc8 was strongly expressed in the trunk anterior and moderately expressed in the trunk posterior; it was not expressed in the brain (Fig. 3). All five of the examined genes, along with $F z d 2$ as a control, exhibited similar expression patterns to those of Hoxc8, although Prkd1 and Ptpn13 were also weakly expressed in brain tissue (Fig. 3 ).

Since Hoxc8 has been reported to be induced in preosteoblastic cells through the TGF- $\beta$ signaling pathway ( $\mathrm{Li}$ et al., 2006; Yang et al., 2000), we tested whether the putative target genes are also concordantly induced along with Hoxc8 in the MC3T3-E1 preosteoblastic cell line. After treating the cells with TGF- $\beta 2$, they were harvested at multiple time points $(0 \mathrm{~h}, 6 \mathrm{~h}$, and $12 \mathrm{~h}$ ), RNA was extracted, and semi-quantitative RT-PCR was performed. As expected, expression of Hoxc8 gradually increased following TGF- $\beta 2$ treatment (Fig. 4). Similarly, four genes of interest, Adam19, Ptpn13, Prkd1, and Tgfbi, were also induced (Fig. 4), although Aldh1a3 was not detected at any time points (data not shown), probably due to gene silencing in MC3T3-E1 cells. Expression of the selected genes, except 


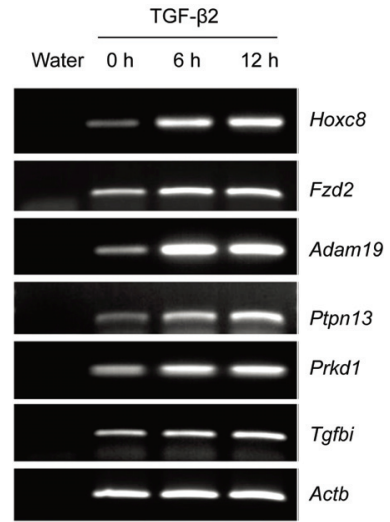

Fig. 4. TGF- $\beta 2$ induces $H o x c 8$ and its putative target genes. MC3T3-E1 cells were treated with TGF- $\beta 2(10 \mathrm{ng} / \mathrm{ml})$, and total RNA was isolated at the indicated time points $(0-12 \mathrm{~h})$. Semiquantitative RT-PCR was then performed to determine the expression patterns of $\mathrm{Hoxc} 8$ and its putative target genes. Fzd2 and Actb were also analyzed as a positive control for Hoxc8 downstream target gene expression and as an internal control, respectively. "Water" represents a negative control reactions containing water instead of template DNA.

Aldh1a3, was confirmed to be positively correlated with Hoxc8 in mouse embryonic tissues and cell lines, in which the TGF- $\beta$ signaling pathway was activated.

\section{Hoxc8 positively regulates expression of the selected genes in vitro}

To further confirm the correlation between Hoxc8 and expression of the selected genes, overexpression and knockdown conditions of Hoxc8 were generated in MC3T3-E1 preosteoblastic cells using a Hoxc8 expression vector (pCDNA3.1Hoxc8) and siRNA against $\mathrm{Hoxc8}$, respectively. When Hoxc8 was transiently overexpressed through introduction of the Hoxc8 expression vector into MC3T3-E1 cells (Fig. 5A), the expressions of Adam19, Ptpn13, Prkd1, and Tgfbi were concordantly increased. Likewise, the expression levels of the four genes decreased when Hoxc8 was knocked down (Fig. 5A). We also stably transfected the Hoxc8 expression vector into C3H10T1/2 and NIH3T3 cell lines, which lack any endogenous expression of Hoxc8. The basal expression levels of Adam19, Ptpn13, Prkd1, and Tgfbi were increased in the Hoxc8expressing cell lines (Fig. 5B). Exceptionally, Aldh1a3 was downregulated upon overexpression of Hoxc8 in C3H10T1/2 cells.

\section{DISCUSSION}

Microarray technology, in combination with chromatin immunoprecipitation (ChIP), has greatly enhanced the discovery of transcription factor target genes (Lei et al., 2005; 2006). Nonetheless, identification of Hox target genes, including Hoxc8, has yet to reach a point that would facilitate delineating their individual roles in specifying the identity of body segments, cell differentiation, migration, and proliferation (Hueber and Lohmann, 2008). Low DNA binding sequence specificity, contextdependent activation or repression, and unknown cofactors present extensive challenges for identification of Hox target genes. These potential pitfalls spurred us to undertake alterna-
A

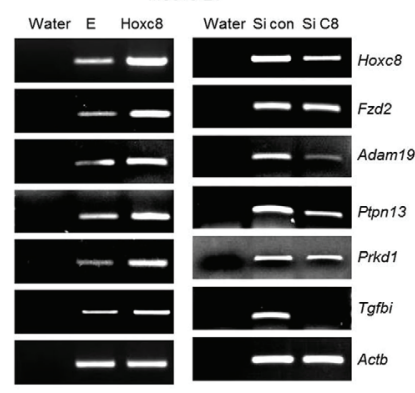

B

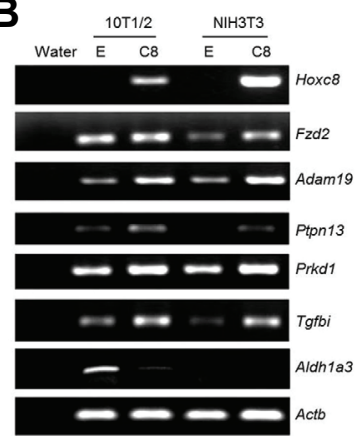

Fig. 5. The effects of Hoxc8 overexpression or knockdown on putative target gene expression. (A) MC3T3-E1 cells were transfected with either empty (pcDNA3.1; E) or Hoxc8 expression vector (pcDNA3.1-Hoxc8; Hoxc8) and harvested $48 \mathrm{~h}$ after transfection (left panel). Total RNA was isolated, and semi-quantitative RT-PCR was performed with the specific primers described in Supplementary Table 1. MC3T3-E1 cells were transfected with control (con) or Hoxc8 siRNA (si C8) and harvested $60 \mathrm{~h}$ after transfection (right panel). Fzd2 and Actb were analyzed as controls. (B) C3H10T1/2 (10T1/2) and NIH3T3 cells were transfected with either empty (pcDNA3.1; E) or Hoxc8 expression vector (C8) and cultured in the selection media containing $500 \mu \mathrm{g} / \mathrm{ml} \mathrm{G} 418$. Total RNA was isolated from stably transfected cell lines, and semi-quantitative RT-PCR was performed. Fzd2 and Actb were analyzed as a positive control for Hoxc8 downstream target gene expression and as an internal control, respectively. "Water" represents a negative control reactions containing water instead of template DNA.

tive methods for identifying Hox target genes. Genes that show similar expression patterns in multiple independent microarray datasets are considered to be highly functionally correlated (Lee et al., 2004; Price and Rieffel, 2004). Therefore, we hypothesized that genes coexpressed with Hoxc8 might suggest plausible roles for Hoxc8, and that they might include cofactors or downstream target genes. In this study, 567 genes were found to be coexpressed with Hoxc8 in at least four out of eight datasets; 23 genes were determined to be coexpressed in six datasets. Among these, genes associated with ECM and cell adhesion were most significantly enriched (Fig. 2 and Supplementary Table S2), irrespective of the tissue types studied. Since the functions of known Hoxc8 target genes are biased toward cell adhesion, it is not surprising that most significantly enriched genes are associated with ECM or cell adhesion. During vertebrate development, Hox genes are expressed during gastrulation, when epithelial mesenchymal transition is initiated at the midline of the embryos, the so-called primitive streak, in order to produce a new class of cells (mesodermal cells) between the epiblasts and hypoblasts. Eventually, Hox genes regulate morphogenesis, during which ECM and cell adhesion molecules play critical roles. Consequently, it is possible that ECM and/or cell adhesion molecules could be major targets of Hox proteins. Among the ECM genes and/or cell adhesion molecules identified (Supplementary Table S2), half of them are ECM structural constituents, like collagen, fibrillin, fibulin, and laminin. Interactions between ECM and cell adhesion molecules regulate cell migration and differentiation in cooperation with growth factors and hormones (Daley and Yamada, 2013). Enrichment of ECM genes has been reported as a significant feature of each dataset analyzed (LeDouxe et 
al., 2006; Li et al., 2007; Timmons et al., 2007). For example, Hoxc8 was overexpressed in white adipocytes compared to brown adipocytes (GDS2743) and in intestinal mesenchymal cells compared to epithelial cells (GDS2699). The transcriptomes of white adipocytes and mesenchymal cells were specifically enriched with ECM genes (Li et al., 2007; Timmons et al., 2007). Therefore, direct or indirect regulation of ECM genes might be a good signature of Hoxc8 activation.

In addition to ECM genes, significant enrichment of transcriptional regulators is also noteworthy. These genes include known Hox cofactors, transcription factors, and chromatin remodeling factors (Supplementary Table S2). The known Hox cofactors, such as the Pbx (Pbx1 and Pbx3) and Meis (Meis1 and Meis2) classes of TALE (Three Amino acid Loop Extension) homeodomain proteins (Moens and Selleri, 2006) suggest that Hoxc8 transactivates downstream target genes by interacting with these cofactors, as other Hox proteins do to overcome poor sequence specificity. Previously, our group identified Pcna, which harbors both $\mathrm{Pbx} 1$ and Hoxc8 binding sites in the promoter region, as a Hoxc8 target gene (Min et al., 2010). Other interesting genes analyzed here are those with homeodomains, such as Zhx1 and Prrx1, which function as transcriptional repressors. Zhx1 was found to interact with DNA methyltransferase (DNMT) 3B (Kim et al., 2007). Interestingly, Prrx $1^{-1}$ mice showed limb bud and skeletal losses (Mann, 1995), and adipogenesis was inhibited (Du et al., 2013). Given that mutations in cofactors result in similar loss-of-function phenotypes of Hox (Moens and Selleri, 2006), Prrx1 might interact with Hoxc8 as a cofactor to repress genes during morphogenesis and adipogenesis. We also identified many other transcription factors that play important roles in embryonic development as Hoxc8 companion genes. This finding was not observed in a previous in vitro cell line experiment (Lei et al., 2006). However, about 50\% of known Hox target genes have been reported to be transcription factors in Drosophila (Hueber and Lohmann, 2008). Thus, it is possible that Hoxc8 transactivates or represses other transcription factors, which in turn amplify signaling and construct Hoxc8-mediated regulatory networks.

To validate the genes analyzed in silico, five genes (Tgfbi, Ptpn13, Prkd1, Adam19, and Aldh1a3) among the 567 were selected at random, and their concordant expression patterns were analyzed with $\mathrm{Hoxc} 8$. We confirmed that their expression levels were positively correlated with that of Hoxc8 in vivo in embryonic tissues and in vitro in cell lines. As an in vitro model, we used a TGF- $\beta$-induced system in MC3T3-E1 cells and overexpression/ knockdown in different cell lines. Therein, we showed that four of the selected genes (Tgfbi, Ptpn13, Prkd1, Adam19), except Aldh1a3, are coexpressed with Hoxc8 and positively regulated by Hoxc8. This allowed us to make sure that Aldh1a3 is, as we expected (coexpressed with Hoxc8 in 3 out of 8 datasets), less relevant to Hoxc8 network, whereas the other four genes, which showed coexpression in at least four of the eight datasets, sufficiently met the requirements to support our in silico methods. Evolutionally conserved Hoxc8 binding sites were found to be present in the promoter regions of the four genes (Supplementary Table S5). Practically, enrichment of Hoxc8 on Hoxc8 binding sites in Adam19 and Ptpn13 was confirmed by ChIP assays (Supplementary Fig. S2), suggesting that the list of genes reported by us include direct target genes. Based on the results, we assume that these genes can be directly or indirectly regulated by Hoxc8 and functionally linked. Especially, Adam19 and Tgfbi, whose functions are associated with ECM and cell adhesion, are likely to be Hoxc8 target genes. Adam19 is a membrane-anchored glycoprotein that plays key roles in embryo implantation, neurogenesis, cardiovascular morphogenesis, and the release of proteins, such as epidermal growth factor receptor ligands and osteoprotegerin ligand, a protein important in osteoclast differentiation and mammary gland development (Fata et al., 2000; Kong et al.,1999; Qi et al., 2009). Tgfbi is a secreted protein that is induced by treatment with transforming growth factor- $\beta$ and inhibits cell attachment (Skonier et al., 1994). It is strongly expressed in the mesenchyme of numerous collagen-rich tissues throughout all stages of murine development (Ferguson et al., 2003; Schorderet et al., 2000), implicating its role in tissue morphogenesis. Together, our collective results suggest that the listed Hoxc8 companion genes are a worthy resource for exploration of Hoxc8 target genes and regulatory networks.

Note: Supplementary information is available on the Molecules and Cells website (www.molcells.org).

\section{ACKNOWLEDGMENTS}

We thank Dr. Jogeswa Gadi for critical reviews and helpful suggestions. This work was supported by the Basic Science Research Program (NRF-2013R1A1A2008399 and NRF2014R1A1A2056986) through the National Research Foundation, Republic of Korea, and partly by a faculty research grant (6-20140147) from Yonsei University College of Medicine, Korea.

\section{REFERENCES}

Chung, H.J., Lee, J.Y., Deocaris, C.C., Min, H., Kim, S.H., and Kim, M.H. (2010). Mouse homologue of the schizophrenia susceptibility gene ZNF804A as a target of Hoxc8. J. Biomed. Biotechnol. 2010, 231708.

Daley, W.P., and Yamada, K.M. (2013). ECM-modulated cellular dynamics as a driving force for tissue morphogenesis. Curr. Opin. Genet. Dev. 23, 408-414.

Du, B., Cawthorn, W.P., Su, A., Doucette, C.R., Yao, Y., Hemati, N., Kampert, S., McCoin, C., Broome, D.T., Rosen, C.J., et al. (2013) The transcription factor paired-related homeobox 1 (Prrx1) inhibits adipogenesis by activating transforming growth factorbeta (TGFbeta) signaling. J. Biol. Chem. 288, 3036-3047.

Fata, J.E., Kong, Y.Y., Li, J., Sasaki, T., Irie-Sasaki, J., Moorehead, R.A., Elliott, R., Scully, S., Voura, E.B., Lacey, D.L., et al. (2000). The osteoclast differentiation factor osteoprotegerin-ligand is essential for mammary gland development. Cell 103, 41-50.

Ferguson, J.W., Mikesh, M.F., Wheeler, E.F., and LeBaron, R.G. (2003). Developmental expression patterns of Beta-ig (betalGH3) and its function as a cell adhesion protein. Mech. Dev. 120, 851-864

Hueber, S.D., and Lohmann, I. (2008). Shaping segments: Hox gene function in the genomic age. Bioessays 30, 965-979.

Hullinger, T.G., Pan, Q., Viswanathan, H.L., and Somerman, M.J. (2001). TGF beta and BMP-2 activation of the OPN promoter: roles of smad- and hox-binding elements. Exp. Cell Res. 262, 6974.

Kikugawa, T., Kinugasa, Y., Shiraishi, K., Nanba, D., Nakashiro, K., Tanji, N., Yokoyama, M., and Higashiyama, S. (2006). PLZF regulates $\mathrm{Pbx} 1$ transcription and $\mathrm{Pbx} 1-\mathrm{HoxC} 8$ complex leads to androgen-independent prostate cancer proliferation. Prostate 66, 1092-1099.

Kim, S.H., Park, J., Choi, M.C., Kim, H.P., Park, J.H., Jung, Y., Lee, J.H., Oh, D.Y., Im, S.A., Bang, Y.J., et al. (2007). Zinc-fingers and homeoboxes 1 (ZHX1) binds DNA methyltransferase (DNMT) 3B to enhance DNMT3B-mediated transcriptional repression. Biochem. Biophys. Res. Commun. 355, 318-323.

Kong, Y.Y., Yoshida, H., Sarosi, I., Tan, H.L., Timms, E., Capparelli, C., Morony, S., Oliveira-dos-Santos. A.J., Van, G., Itie, A., et al. (1999). OPGL is a key regulator of osteoclastogenesis, lymphocyte development and lymph-node organogenesis. Nature 397, 315323.

Kruger, C., and Kappen, C. (2010). Expression of cartilage 
developmental genes in Hoxc8- and Hoxd4-transgenic mice. PLoS One 5, e8978.

Kwon, Y., Ko, J.H., Kim, B.G., and Kim, M.H. (2003). Analysis of plausible downstream target genes of Hoxc8 in F9 teratocarcinoma cells. Mol. Biol. Rep. 30, 141-148.

Kwon, Y., Shin, J., Park, H.W., and Kim, M.H. (2005). Dynamic expression pattern of Hoxc8 during mouse early embryogenesis. Anat. Rec. A Discov. Mol. Cell Evol. Biol. 283, 187-192.

Ladam, F., and Sagerström, C.G. (2014). Hox regulation of transcription: More complex(es). Dev. Dyn. 243, 4-15

LeDoux, M.S., Xu, L., Xiao, J., Ferrell, B., Menkes, D.L., and Homayouni, R. (2006). Murine central and peripheral nervous system transcriptomes: comparative gene expression. Brain Res. $1107,24-41$

Le Mouellic, H., Lallemand, Y., and Brûlet, P. (1992). Homeosis in the mouse induced by a null mutation in the Hox-3.1 gene. Cell $69,251-264$

Lee, H.K., Hsu, A.K., Sajdak, J., Qin, J., and Pavlidis, P. (2004). Coexpression analysis of human genes across many microarray data sets. Genome Res. 14, 1085-1094.

Lei, H., Wang, H., Juan, A.H., and Ruddle, F.H. (2005) The identification of Hoxc8 target genes. Proc. Natl. Acad. Sci. USA $102,2420-2424$

Lei, H., Juan, A.H., Kim, M.S., and Ruddle, F.H. (2006). Identification of a Hoxc8-regulated transcriptional network in mouse embryo fibroblast cells. Proc. Natl. Acad. Sci. USA 103, 10305-10309.

Li, X., Nie, S., Chang, C., Qiu, T., and Cao, X. (2006). Smads oppose Hox transcriptional activities. Exp. Cell Res. 312, 854-864.

Li, X., Madison, B.B., Zacharias, W., Kolterud, A., States, D., and Gumucio, D.L. (2007). Deconvoluting the intestine: molecular evidence for a major role of the mesenchyme in the modulation of signaling cross talk. Physiol. Genomics 29, 290-301.

Mann, R.S. (1995). The specificity of homeotic gene function. Bioessays 17, 855-863.

Mann, R.S., and Chan, S.K. (1996). Extra specificity from extradenticle: the partnership between HOX and PBX/EXD homeodomain proteins. Trends Genet. 12, 258-262.

Mann, R.S., and Affolter, M. (1998). Hox proteins meet more partners. Curr. Opin. Genet. Dev. 8, 423-429.

Min, H., Lee, J.Y., Bok, J., Chung, H.J., and Kim, M.H. (2010). Proliferating cell nuclear antigen (Pcna) as a direct downstream target gene of Hoxc8. Biochem. Biophys. Res. Commun. 392, 543-547.

Min, H., Lee, J.Y., and Kim, M.H. (2012). Structural dynamics and epigenetic modifications of Hoxc loci along the anteroposterior body axis in developing mouse embryos. Int. J. Biol. Sci. 8, 802810.

Min, H., Lee, J.Y., and Kim, M.H. (2013). Hoxc gene collinear expression and epigenetic modifications established during embryogenesis are maintained until after birth. Int. J. Biol. Sci. 9,
960-965

Moens, C.B., and Selleri, L. (2006). Hox cofactors in vertebrate development. Dev. Biol. 291, 193-206.

Pearson, J.C., Lemons, D., and McGinnis, W. (2005). Modulating Hox gene functions during animal body patterning. Nat. Rev. Genet. 6, 893-904.

Price, M.N., and Rieffel, E. (2004). Finding coexpressed genes in counts-based data: an improved measure with validation experiments. Bioinformatics 20, 945-952.

Qi, B., Newcomer, R.G., and Sang, Q.X. (2009). ADAM19/ adamalysin 19 structure, function, and role as a putative target in tumors and inflammatory diseases. Curr. Pharm. Des. 15, 23362348.

Ruthala, K., Gadi, J., Lee, J.Y., Yoon, H., Chung, H.J., and Kim, M.H. (2011). Hoxc8 downregulates Mgl1 tumor suppressor gene expression and reduces its concomitant function on cell adhesion. Mol. Cells 32, 273-279.

Schorderet, D.F., Menasche, M., Morand, S., Bonnel, S., Büchillier, V., Marchant, D., Auderset, K., Bonny, C., Abitbol, M., and Munier, F.L. (2000). Genomic characterization and embryonic expression of the mouse Bigh3 (Tgfbi) gene. Biochem. Biophys. Res. Commun. 274, 267-274.

Shi, X., Yang, X., Chen, D., Chang, Z., and Cao, X. (1999). Smad1 interacts with homeobox DNA-binding proteins in bone morphogenetic protein signaling. J. Biol. Chem. 274, 1371113717.

Shimamoto, T., Tang, Y., Naot, Y., Nardi, M., Brulet, P., Bieberich C.J., and Takeshita, K. (1999). Hematopoietic progenitor cell abnormalities in Hoxc-8 null mutant mice. J. Exp. Zool. 283, 186193.

Skonier, J., Bennett, K., Rothwell, V., Kosowski, S., Plowman, G., Wallace, P., Edelhoff, S., Disteche, C., Neubauer, M., Marquardt, $\mathrm{H}$., et al. (1994). beta ig-h3: a transforming growth factor-betaresponsive gene encoding a secreted protein that inhibits cell attachment in vitro and suppresses the growth of $\mathrm{CHO}$ cells in nude mice. DNA Cell Biol. 13, 571-584.

Timmons, J.A., Wennmalm, K., Larsson, O., Walden, T.B., Lassmann, T., Petrovic, N., Hamilton, D.L., Gimeno, R.E., Wahlestedt, C., Baar, K., et al. (2007). Myogenic gene expression signature establishes that brown and white adipocytes originate from distinct cell lineages. Proc. Natl. Acad. Sci. USA 104, 4401-4406.

Tiret, L., Le Mouellic, H., Maury, M., and Brûlet, P. (1998). Increased apoptosis of motoneurons and altered somatotopic maps in the brachial spinal cord of Hoxc-8-deficient mice. Development 125, 279-291.

Yang, X., Ji, X., Shi, X., and Cao, X. (2000). Smad1 domains interacting with Hoxc-8 induce osteoblast differentiation. J. Biol. Chem. 275, 1065-1072.

Yueh, Y.G., Gardner, D.P., and Kappen, C. (1998). Evidence for regulation of cartilage differentiation by the homeobox gene Hoxc-8. Proc. Natl. Acad. Sci. USA 95, 9956-9961. 